\title{
Chemotherapy induced neutropenia at 1-month mark is a predictor of overall survival in patients receiving TAS-102 for refractory metastatic colorectal cancer: a cohort study
}

Pashtoon M. Kasi ${ }^{1{ }^{*}}$, Daisuke Kotani ${ }^{2+}$, Michael Cecchini ${ }^{3}$, Kohei Shitara ${ }^{2}$, Atsushi Ohtsu ${ }^{2}$, Ramesh K. Ramanathan ${ }^{4}$, Howard S. Hochster ${ }^{3}$, Axel Grothey ${ }^{1}$ and Takayuki Yoshino ${ }^{2}$

\begin{abstract}
Background: TAS-102 (trifluridine and tipiracil hydrochloride; a novel combination oral nucleoside anti-tumor agent) has recently received regulatory approval for patients with refractory metastatic colorectal cancer (mCRC). Internal review of data at a single-institution showed a trend towards better overall survival (OS) for patients who experienced chemotherapy-induced neutropenia at 1-month (CIN-1-month). To explore this finding further, a cohort study was designed based on outcome data from three centers in United States and one from Japan.
\end{abstract}

Methods: CIN-1-month after starting TAS-102 was defined by the Common Terminology Criteria for Adverse Events (CTCAE), version 4.03 as a neutrophil count decrease of $\geq$ grade 2 (absolute neutrophil count $<1500 / \mathrm{mm}^{3}$ ). Patients had confirmed mCRC that was refractory to standard therapies. Patient demographics and clinical characteristics were compared between patients with CIN-1-month (CIN-1-month positive) versus those who did not have CIN-1month (CIN-1-month negative); with the median progression-free survival (PFS) and OS were calculated using the Kaplan-Meier method, and differences evaluated using the Log-rank test.

Results: Our cohort study had a total of 149 patients with data regarding their neutrophil assessment at 1-month mark. Patients who developed $\geq$ grade 2 CIN-1-month had a both longer PFS (median 3.0 months versus 2. 4 months; Log-rank $P$-value $=0.01$ ), as well as OS (14.0 versus 5.6 months; Log-rank $P$-value $<0.0001)$. Only CIN-1month (adjusted HR: 0.21 (95 \% Cl: 0.11-0.38) and higher baseline CEA levels (adjusted HR: 2.00 (95 \% Cl: 1.22-3.35) were noted to be independent predictors of OS. Furthermore, the CIN-1-month was noted to be a statistically significantly predictor of OS over a wide range of cutoffs.

Conclusions: Our observations are novel and hypothesis generating. Neutropenia after starting TAS-102 was associated with better prognosis in patients with refractory mCRC. It can be postulated that the dosage of TAS-102 potentially may need to be increased to achieve better outcomes in patients not experiencing any neutropenia. Further pharmacologic investigations should help elucidate these issues.

Keywords: TAS-102, Colorectal Cancer, Chemotherapy induced neutropenia, Hematological toxicity, Biomarker, Predictive biomarker, Prognostic marker, Pharmacogenomics

\footnotetext{
* Correspondence: kasi.pashtoon@mayo.edu

${ }^{\dagger}$ Equal contributors

'Division of Medical Oncology, Mayo Clinic, 200 First St SW, Rochester 55905,

MN, USA

Full list of author information is available at the end of the article
} 


\section{Background}

TAS-102 (trifluridine and tipiracil hydrochloride; a novel combination oral nucleoside anti-tumor agent) was first approved in Japan in March 2014 and received US Food and Drug Administration (FDA) approval in September 2015 after an international phase-III clinical trial in patients with refractory metastatic colon cancer demonstrated a benefit in overall survival for TAS-102 compared with placebo [1]. Prior to the FDA approval, patients had access to an expanded access program (EAP) of TAS-102 at various institutions within United States. Internal review of outcome data at Mayo Clinic in patients who were treated through the EAP showed a trend towards longer progression free survival (PFS) and overall survival (OS) for patients who were noted to have neutropenia after one cycle (4 weeks) of therapy. To explore this finding further, we validated these findings with outcomes data on additional patients who had received TAS-102 at the Yale Cancer Center, United States, as well as at the National Cancer Center Hospital East, Japan, where the drug had been approved in 2014.

Chemotherapy induced neutropenia (CIN) at 1-month mark [CIN-1-month] after starting TAS-102 was defined by the Common Terminology Criteria for Adverse Events (CTCAE), version 4.03 as a neutrophil count decrease of $\geq$ grade 2 (absolute neutrophil count $<1500 / \mathrm{mm}^{3}$ ). Our hypothesis was that the hematological toxicity (CIN-1-month) was a predictive marker of outcomes in patients with refractory metastatic colorectal cancer through several potential mechanisms (Fig. 1). In fact, in a recently published preclinical model, trifluridine (TFT; which is the anti-tumor component of TAS-102) incorporated itself in the DNA of the colorectal tumor as well as the DNA of the white blood cell in a dose dependent manner [2]. The highest tolerable TFT concentration was the one that provided the highest anti-tumor activity, with hematological toxicity as a potential surrogate marker for the effectiveness of the drug [2]. If our hypothesis is valid, patients who do not have chemotherapy induced neutropenia should have a higher risk of death. The results would provide further rationale to these observations. It will further elucidate the mechanism of action of the drug responsible for its anti-tumor activity. Furthermore, the clinical observation of CIN-1-month would have multiple potential therapeutic implications [3].

\section{Methods \\ Patients}

Patients enrolled in the EAP cohort were age 18 years or older with confirmed metastatic adenocarcinoma of the colon or rectum, and an Eastern Cooperative Oncology Group (ECOG) performance status of 0 or 1 . Patients needed to have previously progressed during or within 3 months following the last administration of approved standard therapies which must have included fluoropyrimidine, oxaliplatin, irinotecan, bevacizumab or aflibercept and cetuximab or panitumumab if $R A S$ wild-type. Patients who had withdrawn from standard treatment due to unacceptable toxicity warranting discontinuation of treatment and precluding retreatment with the same agent prior to progression of disease were also allowed to enter the EAP. The detail of the eligibility criteria for the EAP that was open at 33 different sites within United States is available at http://clinicaltrials.gov (identifier: NCT02286492) [4]. For the purpose of this study, our EAP cohort comprised of patients from three cancer centers in United States (Mayo Clinic Rochester, Mayo Clinic Arizona and Yale Cancer Center).

\section{Expanded Access Program (EAP) cohort from United States}

Our 'EAP cohort' comprised of patients who were enrolled through the expanded access program (EAP) of TAS-102 at the Mayo Clinic (Rochester and Arizona sites, United

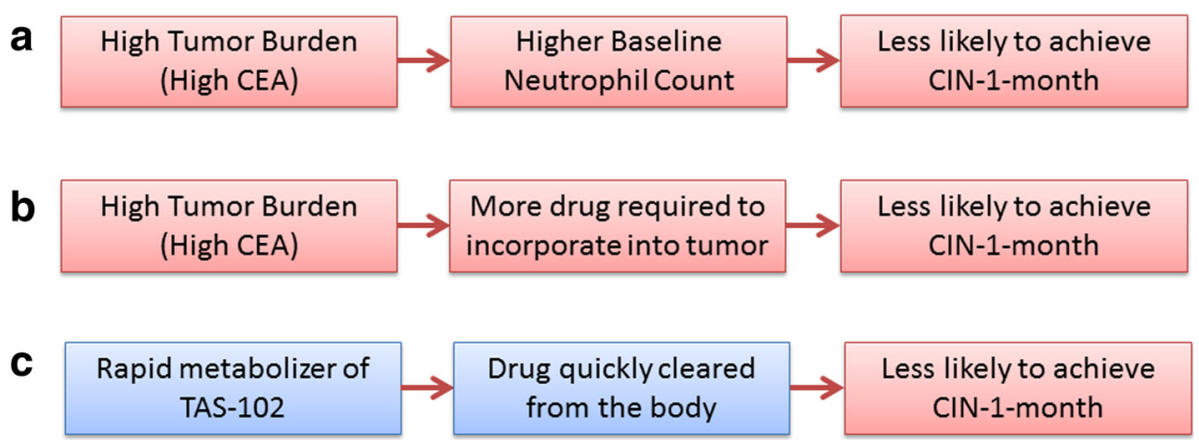

Fig. 1 Postulated mechanisms between association of chemotherapy-induced neutropenia at 1-month mark (CIN-1-month) and overall survival: (a) Firstly, one may postulate that patients with a high tumor burden could have a high baseline neutrophil count; making it less likely to experience CIN-1-month; (b) Secondly, since the drug incorporates into the tumor, for patients with a high tumor burden, it is possible that that the standard dosage of the drug may not be enough to exert myelotoxicity; and c) Finally, individuals experiencing different degrees of neutropenia may have different pharmacokinetics of TAS-102 and its metabolites 
States) and the Yale Cancer Center, New Haven, Connecticut, United States between March 04, 2015 and September 30, 2015. Institutional Review Board (IRB) approvals were obtained from both institutions prior to the initiation of the EAP, alongside approval from Taiho Pharmaceutical Co. LTD.

\section{Validation cohort from Japan}

Our 'validation cohort' included patients with histologically confirmed colorectal adenocarcinoma who were treated with TAS-102 in Japan from May 01, 2014 to September 30, 2015 [5]. The retrospective data from the validation cohort was collected under an IRB waiver in accordance with the Japanese Ethical Guidelines for Epidemiological Research.

\section{Study design}

To answer the question if CIN-1-month affects outcomes in patients with refractory metastatic colorectal cancer, a cohort study was performed. All patients were treated with TAS-102 at a dose of $35 \mathrm{mg} / \mathrm{m}^{2}$ administered orally twice daily for 5 days a week with 2 days' rest for 14 days, followed by a 14-day rest (1 treatment cycle). Patients with chemotherapy induced neutropenia (CIN) at 1-month mark [CIN-1-month] after starting TAS-102 as defined by the Common Terminology Criteria for Adverse Events (CTCAE), version 4.03 as a neutrophil count decrease of $\geq$ grade 2 (absolute neutrophil count $<1500 / \mathrm{mm}^{3}$ ) were defined as the CIN-1-month positive group. Patients without CIN-1-month were the reference group (CIN-1-month negative group). Patients were subsequently assessed for the different outcomes as described.

\section{Endpoints and assessments}

The medical records of patients were retrospectively reviewed by investigators at the four institutions and data abstracted for the purposes of this study to gather data regarding PFS, OS and outcome of their first imaging computed tomography (CT) scans. PFS was defined as the interval from the start of TAS-102 treatment to either disease progression or death. OS was defined as the interval from the start of TAS-102 treatment to death. For PFS or OS, the patients were censored at their last follow-up visit if they were free of disease progression or death, respectively [5]. The median PFS and OS were calculated using the Kaplan-Meier method, and differences between patients with and without CIN-1-month were evaluated using the Log-rank test. Data from both cohorts were combined for further reporting and analysis. Additionally, separate subset analyses were also conducted for the endpoints described. Patients with less than 4 weeks (28 days) of follow up were excluded from the final analysis. Statistical analysis was performed using JMP ${ }^{\circ} 10.0$ (2012 SAS Institute Inc.). Laboratory data regarding absolute neutrophil counts and carcinoembryonic antigen (CEA) level were also collected if available at baseline and on day 1 of initiation of different cycle visits. Based on the median, patients were divided into older adults $($ age $>65)$ and young adults (age $\leq 65)$, high CEA levels $(\mathrm{CEA}>55 \mathrm{ng} / \mathrm{ml})$ and low CEA levels $(\leq 55 \mathrm{ng} / \mathrm{ml})$, and high baseline neutrophil count $\left(>4300 / \mathrm{mm}^{3}\right)$ and low baseline neutrophil count $\left(\leq 4300 / \mathrm{mm}^{3}\right)$. Sensitivity analyses were also conducted based on different cutoffs of neutrophil count at 1-month mark to assess the relationship between hematologic toxicity and overall survival.

\section{Results \\ Patients}

The EAP cohort had a total of 83 patients (49 patients from the two Mayo Clinic sites and 34 from the Yale Cancer Center). The validation cohort from Japan had 92 patients. Thus, the study included a total of 175 individuals. Excluding patients with less than 4 weeks (28 days) of follow up (18 patients; $10 \%$ ), our final cohort had a total of 157 patients. Data on neutrophil counts at the 4-week mark were available in 149 patients, 69 (46\%) of which experienced neutropenia (CIN-1-month positive) and 80 (54\%) who did not (CIN-1-month negative).

\section{Comparison between EAP and validation cohorts}

We compared baseline characteristics and outcome data between the EAP cohort from United States and the validation cohort from Japan to see if there were any differences that might be explained by patients from different origins. Patient in the EAP cohort were noted to be younger (median age 61 years versus 65 years; $P$-value $=0.08$ ). No statistically significant differences were noted between the two cohorts in the demographic characteristics or outcomes (data not shown). Therefore, further analyses reported in the paper are on the 2 cohorts combined.

\section{Efficacy}

A total of 144 patients had their first staging imaging scans ( $\sim$ after 2 cycles of therapy) available for review. At first evaluation, $84(58 \%)$ patients had progressive disease (PD), 55 (38\%) patients had stable disease (SD) and $5(4 \%)$ patients had a partial response (PR) to TAS102. The median overall survival (OS) and progressionfree survival (PFS) was 8.9 months and 2.6 months; comparable to the 7.1 month and 2.0 months in the original phase-III study [1]. Detailed data on safety and outcomes of 55 of the 92 patients were recently published by the authors from Japan [5].

\section{Chemotherapy Induced Neutropenia (CIN) - at 1-month mark}

Table 1 summarizes the characteristics of patients with chemotherapy-induced neutropenia at 1 month 
Table 1 Comparison of patients with metastatic colorectal cancer who achieved chemotherapy induced neutropenia (CIN) $\geq$ grade 2 CTCAE (EXPOSED - CIN-1-Month positive) as compared to those who did NOT have $\geq$ grade 2 CIN at the 1-month mark - (Referent - CIN-1-Month - ve) after starting treatment with TAS-102

\begin{tabular}{|c|c|c|c|c|}
\hline & Variable & $\begin{array}{l}\text { Patients with CIN-1-month N (\%) } \\
\text { (CIN-1 month positive) }\end{array}$ & $\begin{array}{l}\text { Patients without CIN-1-month N (\%) } \\
\text { (CIN-1 month - ve) }\end{array}$ & $P$-value \\
\hline 1 & Number & $69(46.3 \%)$ & $80(53.7 \%)$ & \\
\hline 2 & Females & $29(42.0 \%)$ & $42(52.5 \%)$ & 0.20 \\
\hline 3 & Older Adults (Age > 65 years) & $39(56.5 \%)$ & $39(48.8 \%)$ & 0.34 \\
\hline 4 & EAP (versus validation cohort) & $33(47.8 \%)$ & $37(46.3 \%)$ & 0.85 \\
\hline 5 & Primary site Colon (vs. Rectal) & $38(55.1 \%)$ & $50(62.5 \%)$ & 0.36 \\
\hline 6 & RAS-wild type & $32(46.4 \%)$ & $41(51.3 \%)$ & 0.56 \\
\hline 7 & High Baseline CEA (> 55 ng/ml) & $24(34.7 \%)$ & $43(53.8 \%)$ & $0.02^{*}$ \\
\hline 8 & Higher Baseline Neutrophil Count (> 4300/mm³) & 19 (27.5\%) & $55(68.8 \%)$ & $<0.0001^{* *}$ \\
\hline \multirow[t]{4}{*}{9} & Overall disease control rate (DCR) & $32(49.2 \%)$ & $28(37.8 \%)$ & 0.18 \\
\hline & -Partial Response (PR) & $-4(6.1 \%)$ & $-1(1.3 \%)$ & \\
\hline & -Stable Disease (SD) & $-28(43.1 \%)$ & $-27(36.5 \%)$ & \\
\hline & -Progressive Disease (PD) & $-33(50.8 \%)$ & $-46(62.2 \%)$ & \\
\hline 10 & Progression Free Survival (PFS) in months $\left(95 \% \mathrm{Cl}^{2}\right)$ & $3.0(2.3-3.6)$ & $2.4(1.9-2.9)$ & $0.0096^{*}$ \\
\hline 11 & Overall Survival (OS) in months $\left(95 \% \mathrm{Cl}^{2}\right)$ & $14.0\left(11.2-\mathrm{NR}^{1}\right)$ & $5.6(4.7-8.1)$ & $<0.0001^{* *}$ \\
\hline
\end{tabular}

${ }^{1} \mathrm{NR}$ not reached, ${ }^{2} \mathrm{Cl}$ confidence interval

${ }^{*} p$-value $<0.05$

** $p$-value $<0.001$

(CIN-1-month positive) and those who did not achieve chemotherapy-induced neutropenia at 1 month (CIN1-month negative). A total of $69(46.3 \%)$ patients developed $\geq$ grade $2 \mathrm{CIN}$ at 1 month-mark. Patients who developed $\geq$ grade 2 CIN-1-month had a both longer progression-free survival (median 3.0 months versus 2.4 months; Log-rank $P$-value $=0.0096$; Fig. 2 ) as well as overall survival (14.0 versus 5.6 months; Log-rank $P$-value $<0.0001$; Fig. 2). Additionally, the number of CIN-1-month positive patients achieved disease control was $32(49.2 \%)$ as compared to $28(37.8 \%)$ in the CIN-1-month negative group $(P$-value $=0.18)$. There were no significant differences in the sex, older adults, primary site (colon versus rectum) and $R A S$-mutational status between the two cohorts.
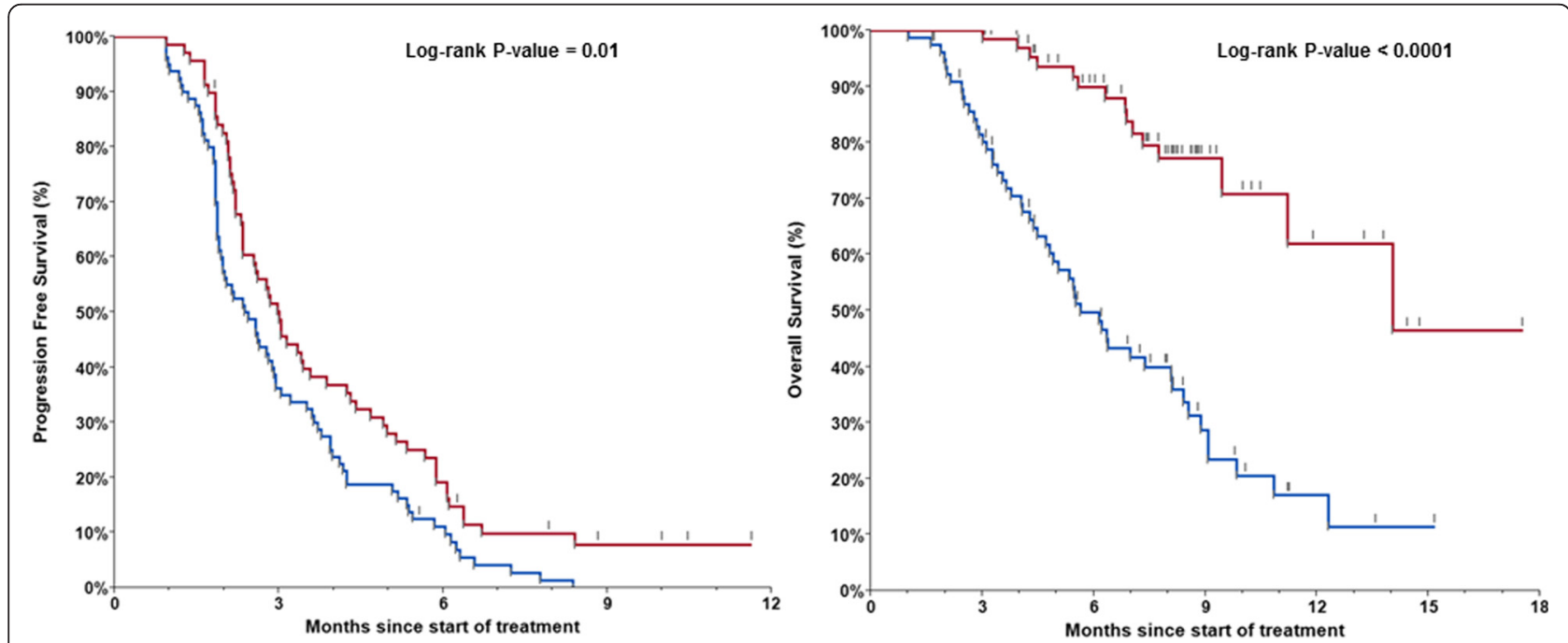

Fig. 2 Kaplan-Meier curves for progression-free survival (PFS; median 3.0 months versus 2.4 months; Log-rank P-value $=0.01$ ) as well as overall survival (OS; median 14.0 months versus 5.6 months; Log-rank P-value < 0.0001) for patients who achieved chemotherapy induced neutropenia $(\mathrm{CIN}) \geq$ grade 2 CTCAE (red line - 69 patients $(46.3 \%)$ - CIN-1-month positive) as compared to those who did NOT have $\geq$ grade 2 CIN at the 1-month mark (blue line - 80 patients (53.7\%) - CIN-1-month negative) after starting treatment with TAS-102 
Hazard ratios (HR) for overall survival alongside $95 \%$ confidence intervals (CI) were estimated through the Cox proportional hazards model (Table 2). Separate analyses for different stratum are shown. Only CIN-1-month (adjusted HR: 0.21; 95 \% CI: 0.11-0.38: $P$-value < 0.0001) and 'Higher Baseline CEA' (adjusted HR: 2.00; 95 \% CI: 1.22-3.35: $P$-value 0.0062) were noted to be independent predictors of overall survival. All $P$-values for interaction were higher than 0.20. Overall survival was significantly different between the CIN-1-month positive and CIN-1month negative patients in both the 'Higher Baseline CEA' (9.4 months versus 4.5 months; $P$-value 0.0006 ) and 'Lower Baseline CEA' (median not reached versus 8.0 months; $P$-value 0.0003) groups of colorectal cancer patients. Furthermore, the CIN-1-month was noted to be a statistically significantly predictor of overall survival over a wide range of cutoffs (Table 2).

\section{Discussion}

Our study suggests that chemotherapy induced neutropenia at 1-month mark (CIN-1-month) after starting TAS-102 appears to be a prognostic and/or predictive biomarker of both PFS and OS in patients with refractory

Table 2 Association between CIN-1-month and overall survival in patients with metastatic colorectal cancer receiving TAS-102

\begin{tabular}{|c|c|c|}
\hline Sample or stratum & $\mathrm{HR}(95 \% \mathrm{Cl})^{\mathrm{a}}$ & $P$-value \\
\hline Overall & $0.22(0.12-0.38)$ & $<0.0001$ \\
\hline \multicolumn{3}{|l|}{ Strata } \\
\hline Men & $0.15(0.06-0.33)$ & $<0.0001$ \\
\hline Women & $0.31(0.13-0.67)$ & 0.0023 \\
\hline Rectal & $0.36(0.15-0.78)$ & 0.0092 \\
\hline Colon & $0.15(0.06-0.34)$ & $<0.0001$ \\
\hline RAS-mutant & $0.32(0.15-0.64)$ & 0.001 \\
\hline RAS-wild type & $0.14(0.05-0.35)$ & $<0.0001$ \\
\hline Older adults (age $>65$ years) & $0.19(0.09-0.40)$ & $<0.0001$ \\
\hline Younger adults (age $\leq 65$ years) & $0.21(0.07-0.52)$ & 0.0003 \\
\hline Higher Baseline CEA (> 55 ng/ml) & $0.26(0.11-0.56)$ & 0.0004 \\
\hline Lower Baseline CEA ( $\leq 55$ ng/ml) & $0.18(0.07-0.42)$ & $<0.0001$ \\
\hline $\begin{array}{l}\text { Higher Baseline Neutrophil Count } \\
\left(>4300 / \mathrm{mm}^{3}\right)\end{array}$ & $0.25(0.08-0.57)$ & 0.0005 \\
\hline $\begin{array}{l}\text { Lower Baseline Neutrophil Count } \\
\left(\leq 4300 / \mathrm{mm}^{3}\right)\end{array}$ & $0.27(0.11-0.65)$ & 0.0035 \\
\hline \multicolumn{3}{|l|}{ Absolute Neutrophil cutoff at 1-month } \\
\hline CIN-1-month $\left(<1000 / \mathrm{mm}^{3}\right)$ & $0.34(0.16-0.66)$ & 0.0008 \\
\hline CIN-1-month $\left(<1500 / \mathrm{mm}^{3}\right)$ & $0.22(0.12-0.38)$ & $<0.0001$ \\
\hline CIN-1-month $\left(<2000 / \mathrm{mm}^{3}\right)$ & $0.28(0.17-0.47)$ & $<0.0001$ \\
\hline CIN-1-month $\left(<2500 / \mathrm{mm}^{3}\right)$ & $0.25(0.16-0.42)$ & $<0.0001$ \\
\hline CIN-1-month $\left(<3000 / \mathrm{mm}^{3}\right)$ & $0.26(0.16-0.43)$ & $<0.0001$ \\
\hline
\end{tabular}

metastatic colorectal cancer. Individuals who developed CIN-1-month had a significant improved survival (14.0 versus 5.6 months; $P$-value $<0.0001)$.

In both the previously conducted phase-II and the recently published phase-III study, neutropenia was the most common adverse event in patients who received TAS-102 [1, 6]. This required at least one dose reduction and/or a treatment interruption in up to a third of patients [7-9]. Similar safety and efficacy was noted in subsequent studies [5].

Although the mechanism underlying the association of CIN-1-month and OS in patients with refractory metastatic colorectal cancer is not entirely clear, three hypotheses can be postulated (Fig. 1). Firstly, one may postulate that patients with a high tumor burden could have a high baseline neutrophil count; making it less likely to experience CIN-1-month. Secondly, since the drug incorporates into the tumor, for patients with a high tumor burden, it is possible that that the standard dosage of the drug may not be enough to exert myelotoxicity. Finally, individuals experiencing different degrees of neutropenia may have different pharmacokinetics of TAS-102 and its metabolites. Our analyses, however, showed that the CIN-1-month was still statistically significantly associated with OS after controlling for tumor burden and other potential confounders.

Based on these observations, one can postulate that the dosage of TAS-102 may need to be increased in patients not experiencing any neutropenia to improve outcomes. Conversely, one may consider increasing the interval of chemotherapy instead of decreasing the TAS-102 dose in the subset of patients having significant decline in their absolute neutrophil counts without any clinical complications [10]. Prophylactic antibiotics and the use of growth factor support with a different dosing schedule may be other considerations, especially when considering combining this novel agent with other chemotherapy regimens for potential future clinical trials [11].

Of note, correlation between chemotherapy induced toxicities and favorable outcomes have been described previously in a number of different settings [12, 13]. Whether this is purely related to pharmacokinetics of the drug or other proposed mechanisms as outlined above remains to be determined.

Our study, however, has several limitations. First, our sample size was relatively small for some of the stratified analyses as shown by wide confidence intervals. Follow up for some of the patients in the EAP cohort is still relatively short. The majority of the patients, however, had already progressed on the study drug given the highly refractory nature of the population under study.

Our observation is hypothesis generating and has a number of strengths. First, it was based on a prospectively enrolled EAP cohort as part of the expanded access 
clinical trial with similar cohort of patients with refractory colorectal cancer. Second, we were able to corroborate the observations in an independent cohort of patients from a different center as well as a different country. Third, analyses were stratified for several known prognostic factors and potential confounding effects were explored. Validation of our findings in an independent population cohort is the strength of this analysis and provides a readily available potentially predictive as well as prognostic biomarker (CIN-1-month) for patients with metastatic colorectal cancer.

\section{Conclusions}

Neutropenia after starting TAS-102 was associated with better prognosis in patients with refractory mCRC. Our findings are clinically relevant and have led to reanalyses of both the initial randomized phase-II (Study J003-10040030) and phase-III (RECOURSE trial) studies of TAS-102 versus placebo, and similar results were seen. These findings are important since it can be postulated that the dosage of TAS-102 potentially may need to be increased to achieve better outcomes in patients not experiencing any neutropenia. Further pharmacologic investigations should help elucidate these issues and help validate the potential utility of CIN-1-month as a prognostic and/or predictive biomarker of TAS-102 for patients with refractory mCRC.

\section{Abbreviations \\ CEA, carcinoembryonic antigen; CIN-1-month, chemotherapy-induced neutro- penia at 1-month; CTCAE, common terminology criteria for adverse events; EAP, expanded access program; mCRC, metastatic colorectal cancer; OS, overall survival; PFS, progression-free survival; TAS-102, (trifluridine and tipiracil hydrochloride; a novel combination oral nucleoside anti-tumour agent)}

\section{Acknowledgements}

We are especially grateful to Daniel J. Sargent, Ph.D. for his expert opinion and guidance during the statistical analyses. Thanks are also due to Taiho Pharmaceutical Co. LTD for allowing us to use the expanded access program (EAP) data on patients receiving TAS-102 in United States.

\section{Funding}

No funding was obtained for this study. Divisional support from Department of Oncology, Mayo Clinic, Rochester was utilized for access to statistical and reference software.

\section{Availability of data and materials}

Merged files with individual data will not be shared since this study reporting the combined analysis of the expanded access program at Mayo Clinic Rochester/Arizona and Yale Cancer Center alongside retrospective data from the validation cohort from National Cancer Center Hospital East, Kashiwa, Chiba, Japan did not specifically seek permission for this purpose.

\section{Authors' contributions}

Authors PMK and AG initially formulated the study design and hypothesis. This was further refined by all the authors. Subsequently, authors PMK, AG, $M C, K S, A O, R K R, H S H, A G$ and TY were involved in data collection at all their individual sites respectively. These were combined and statistical analysis was performed by author PMK alongside active supervision and guidance from DK, AG and TY. All this was revised and refined from input from all the authors. Authors PMK and DK wrote the initial draft. This was extensively revised by all the authors. All the authors approved the final draft for publication. Abstract of our findings were included in the proceedings of the American Society of
Clinical Oncology (ASCO) meeting in Chicago, 2016 (J Clin Oncol Meeting Abstracts May 2016 vol. 34 no. 15_suppl e15124). The minor revisions requested by the reviewers and the editor were done by PMK.

\section{Competing interests}

PMK: None; DK: None; MC: None; KS: Research support from Dainippon Sumitomo Pharma, Lilly, MSD, Sanofi, Daiichi Sankyo, Bayer, Taiho Pharmaceutical, Chugai Pharma and Yakult Inc. Consulting/advisory role for Chugai Pharma, Takeda, Bayer and Lilly Inc.; AO: Research support from Bristol-Myers Squibb; family member in Celgene Inc.; RKR: Research support from Abbvie, Sanofi, Genentech, Bayer, Halozyme, Vaccinex, Celgene, Tekmira, Merck/Schering Plough, Biomarin, Merrimack and Verastem. Consulting/advisory role for Celgene, Lilly, Genentech and Vaccinex; HSH: Speaker's bureau Genomic Health; Consulting/advisory role for Bayer, Boehringer Ingelheim, Genentech, Amgen, Sirtex Medical and Bristol-Myers Squibb; AG: Research support from Genentech, Bayer, Eisai, Pfizer, Eli-Lilly; TY: Research funding from Umitomo Dainippon Pharma Co., Ltd.

\section{Consent for publication}

Not applicable.

\section{Ethics approval and consent to participate}

Institutional Review Board (IRB) approvals were obtained from both institutions (Mayo Clinic Rochester/Arizona and Yale Cancer Center) prior to the initiation of the EAP alongside approval from Taiho Pharmaceutical Co. LTD. The retrospective data from the validation cohort from Japan was collected under an IRB waiver in accordance with the Japanese Ethical Guidelines for Epidemiological Research.

\section{Author details}

${ }^{1}$ Division of Medical Oncology, Mayo Clinic, 200 First St SW, Rochester 55905, MN, USA. ${ }^{2}$ Department of Gastrointestinal Oncology, National Cancer Center Hospital East, Kashiwa, Chiba, Japan. ${ }^{3}$ Division of Medical Oncology, Yale Cancer Center, New Haven, CT, USA. ${ }^{4}$ Division of Medical Oncology, Mayo Clinic, Scottsdale, Arizona, USA.

Received: 14 March 2016 Accepted: 28 June 2016

Published online: 13 July 2016

\section{References}

1. Mayer RJ, Van Cutsem E, Falcone A, et al. Randomized trial of TAS-102 for refractory metastatic colorectal cancer. N Engl J Med. 2015:372(20):1909-19.

2. Yamashita F, Komoto I, Oka H, et al. Exposure-dependent incorporation of trifluridine into DNA of tumors and white blood cells in tumor-bearing mouse. Cancer Chemother Pharmacol. 2015;76(2):325-33.

3. Tanaka N, Sakamoto K, Okabe H, et al. Repeated oral dosing of TAS-102 confers high trifluridine incorporation into DNA and sustained antitumor activity in mouse models. Oncol Rep. 2014;32(6):2319-26.

4. ClinicalTrials.gov. Expanded Access Study of TAS-102 in Patients With Metastatic Colorectal Cancer. 2016; https://clinicaltrials.gov/ct2/show/ NCT02286492. Accessed 15 June 2016.

5. Kotani D, Shitara K, Kawazoe A, et al. Safety and efficacy of trifluridine/ tipiracil monotherapy in clinical practice for patients with metastatic colorectal cancer: experience at a single Institution. Clin Colorectal Cancer. 2015 [Epub ahead of print]

6. Yoshino T, Mizunuma N, Yamazaki K, et al. TAS-102 monotherapy for pretreated metastatic colorectal cancer: a double-blind, randomised, placebo-controlled phase 2 trial. Lancet Oncol. 2012;13(10):993-1001.

7. Doi T, Ohtsu A, Yoshino T, et al. Phase I study of TAS-102 treatment in Japanese patients with advanced solid tumours. Br J Cancer. 2012;107(3):429-34.

8. Hong DS, Abbruzzese $\mathrm{J}$, Bogaard $\mathrm{K}$, et al. Phase I study to determine the safety and pharmacokinetics of oral administration of TAS-102 in patients with solid tumors. Cancer. 2006;107(6):1383-90.

9. Bendell JC, Rosen LS, Mayer RJ, et al. Phase 1 study of oral TAS-102 in patients with refractory metastatic colorectal cancer. Cancer Chemother Pharmacol. 2015;76(5):925-32.

10. Overman MJ, Varadhachary G, Kopetz S, et al. Phase 1 study of TAS-102 administered once daily on a 5-day-per-week schedule in patients with solid tumors. Invest New Drugs. 2008;26(5):445-54. 
11. Doi T, Yoshino T, Fuse N, et al. Phase I study of TAS-102 and irinotecan combination therapy in Japanese patients with advanced colorectal cancer. Invest New Drugs. 2015;33(5):1068-77.

12. Tewari KS, Java JJ, Gatcliffe TA, Bookman MA, Monk BJ. Chemotherapyinduced neutropenia as a biomarker of survival in advanced ovarian carcinoma: an exploratory study of the gynecologic oncology group. Gynecol Oncol. 2014;133(3):439-45.

13. Liu R, Huang M, Zhao X, et al. Neutropenia predicts better prognosis in patients with metastatic gastric cancer on a combined epirubicin, oxaliplatin and 5-fluorouracil regimen. Oncotarget. 2015;6(36):39018-27.

Submit your next manuscript to BioMed Central and we will help you at every step:

- We accept pre-submission inquiries

- Our selector tool helps you to find the most relevant journal

- We provide round the clock customer support

- Convenient online submission

- Thorough peer review

- Inclusion in PubMed and all major indexing services

- Maximum visibility for your research

Submit your manuscript at www.biomedcentral.com/submit
Biomed Central 